\title{
A Study on Military Violence and Suicidal Ideation in South Korea
}

\author{
The Moderating Effect of Parent-Child Positive Communication
}

\author{
[Yoon Hee Choi $\cdot$ Jae Yop Kim]
}

\begin{abstract}
This Study is to examine the effects of verbal or physical violence on suicidal ideation of enlisted soldiers in South Korea. Although the prevalence of military violence and suicidal ideation of soldiers is high, limited research has been carried out on the relationship between military violence and suicidal ideation among them. Data from 1,000 army, navy and air force enlisted soldiers were obtained from a nationwide survey in 2016. Descriptive analysis and Poisson regression analysis were conducted using SPSS 24.0. Due to a skewed distribution of the prevalence of suicidal ideation, this study applied a zero-inflated Poisson (ZIP) model, which has been shown to address issues of skewed count variables. The results showed that 175 soldiers $(17.6 \%)$ were revealed to be the victims of verbal or physical violence. At the same time, $17.9 \%$ of military violence victims experienced suicidal ideation, whereas $4.2 \%$ of non-victimized soldiers had suicidal ideation. Although military violence had statistically relationship with suicidal problem among the victimized soldiers, further analysis showed that parent-child positive communication worked as a moderator between military violence and suicidal ideation $(\mathrm{B}=-.586, \mathrm{SE}=.263, \mathrm{p}<.001)$. Results suggest that the pragmatic and political interventions are needed to reduce military violence. Intervention strategies to address the victimized soldiers' suicidal ideation are discussed as well. (Abstract)
\end{abstract}

Keywords-military social work, enlisted soldier, military violence, suicidal ideation, parent-child positive communication

Yoon Hee Choi (Author)

Graduate School of Social Welfare of Yonsei

Republic of Korea

Jae Yop Kim (Author)

Department of Social Welfare, Yonsei University

Republic of Korea 